\title{
Integration of the ROS Framework in Soccer Robotics: The NAO Case
}

\author{
Leonardo Leottau Forero, José Miguel Yáñez, and Javier Ruiz-del-Solar \\ Advanced Mining Technology Center, Department of Electrical Engineering, \\ Universidad de Chile, \\ Av. Tupper 2007, Santiago, Chile \\ \{dleottau, jruizd\}@ing.uchile.cl, jm.yanez.arancibia@gmail.com \\ http://uchilert.amtc.cl
}

\begin{abstract}
The SPL robot soccer league focuses its efforts on the development of robot control software for standard humanoid robots. Nevertheless, few interchange of software modules are observed in the league, being the B-Human effort an exception. In addition, a large difference in performance is observed between experienced teams and new teams. This situation makes difficult the incorporation of new teams in the league. Therefore, it seems attractive to explore the use of ROS within the SPL soccer robotics community in order to revert the described situation. As a first step, this paper presents some work in this direction, such as the installation of ROS in the new NAO V4 robots, the integration of the B-Human motion engine as a ROS node, and the communication of two robots running a ROS-based control software.
\end{abstract}

Keywords: Humanoid robots, NAO robot, Robot Operating System (ROS), shared code.

\section{Introduction}

The SPL (Standard Platform League) is a RoboCup robot soccer league in which all teams compete with identical robots; since 2008 the robots being used are the Aldebaran NAO robots [5. The research efforts focus on the development of sophisticated control software for fully autonomous operation of a team of robots. With teams participating in the league for 5-10 years, or ever more, some control software solutions have reached a very high level of effectiveness and functionality, which new teams cannot reach easily. In addition, even tough being a league where most of the work is on the development of software, there are very few cases of code sharing. One of these cases is the one of the B-Human team [1, which makes every year its software open to the community. However, even in this case it is not easy to use just some specific parts of the code. Normally teams use the whole code and modify the high-level behaviors. In addition, it is not easy for other teams to develop new components that can be integrated in the B-Human software. Basically, the B-Human software is being developed and extended just by the B-Human team.

S. Behnke et al. (Eds.): RoboCup 2013, LNAI 8371, pp. 664-671, 2014.

(C) Springer-Verlag Berlin Heidelberg 2014 
On the other hand, the ROS (Robot Operating System) Framework, which can be defined as an open-source, meta-operating system for robots, has emerged as tool that allows the sharing of robot software modules. In addition to provide a proper architecture to build robot applications and to communicate appropriately different modules using the ROS Node concept, the main attractiveness of ROS is that is being used almost by everyone in the robotics research community. In this context it seems attractive to explore the use of ROS within the SPL soccer robotics community. The use of ROS within the SPL soccer community could allow:

- to share easily software module between teams,

- to encourage the development of very specialized solutions which can be shared among the teams,

- to facilitate the incorporation of new teams in the league,

- to attract new students and new researchers to the leagues teams,

- to encourage the specialization of some teams in some robot control areas (e.g. motion control or perception), and

- to facilitate the comparison and benchmarking of specific software modules.

In order to explore the use of ROS within the SPL league, the first step requires being able to compile ROS in the NAO robots, and to build a basic architecture for these robots. The main goal of this paper is to present such developments. The article is organized as follows: Section 2 describes the use of the ROS framework in the NAO robots. This includes a description of how to install ROS into the NAO robots and the integration of the B-Human Motion Engine as a ROS Node. Section 3 presents as a proof of concept, the communication between two NAO robots using a ROS-based core. Finally, some conclusions are drawn in section 4

\section{ROS Use in NAO Robots}

\subsection{ROS Installation on NAO Robots}

There are few research groups and labs working with NAO robots and ROS [3] 7] 4. The Humanoid Robots Lab at the Albert-Ludwigs-Unversitaet that have developed the nao-robot stack which contains some useful nodes to integrate the NAO robot into ROS [8]. Within its activities of robotic soccer, the Mobile Robotics Group of the University of Chile is currently using the ROS framework as part of their NAO robot software architecture [3]. Recently the group has uploaded to the ROS community a detailed tutorial to build, install and run ROS natively onto the NAO V4 6]. To the best on our knowledge, this is the first tutorial that provides a step-by-step guide to build, install and run ROS Fuerte embedded onto the Atom CPU of the latest NAO robot. In Figure 1 are shown the main steps of this installation. 


\section{Requirements}

- Download and uncompress the appropriate Naoqi toolchain version.

- Download ROS BASE from source code.

- Setup paths, environment variables, and directories such as: ROS-NAO root path (\$TARGETDIR), ROS fuerte installation path, the CTC directory where Aldebaran's cross-compilation toolchain was uncompressed, the CTC boost root path, among others.

\section{Preliminary Steps}

- To include NAO's toolchain within ROS build toolchain.

- Since NAO's toolchain does not contains "log4cxx.so" nor "aprutil*.so" libraries, these should be copied.

\section{Compiling ROS Base from source}

- To compile ROS Base from source, generating the project using cmake, including the Aldebaranś Nao Cross toolchain withing the ROS toolchain file. It is important to set the CMake-Install-Path in the same absolute path where ROS will be run in the Nao`s CPU.

\section{Copying some files, scripts and missing libraries}

- It is necessary to copy some files, scripts and libraries that are missing on the NAO. These files are needed for launching ROS on the NAO's CPU. For example the log4cxx, boost and apr-util libraries, python packages "yaml”, among others.

\section{Installing and running ROS onto NAO's CPU}

- To install ROS in the NAO simply copy the installation root folder to the robot via ssh, remembering that the \$TARGETDIR necessarily must match the installation path indicated in the robot (/home/nao/ros-nao for example).

- After that it is necessary to specify to ROS the path where files, scripts and libraries were added in the previous step. This should be done by exporting the environment variables \$LD_LIBRARY_PATH and \$PYTHONPATH. These paths indicate to ROS an alternative directory to find libraries and python files respectively. It must be set on each terminal where some ROS task will be run.

- To run ROS onto the Nao`s CPU, it is necessary run roscore command and executes each needed process-node.

Fig. 1. ROS installation procedure in NAO V4 robots. A detailed tutorial can be found in [6]. 


\subsection{Integration of the B-Human Walking and Motion Engine as a ROS Node}

Currently, UChile Robotics Team $[\underline{3}$ is using the B-Human walking and motion engine [2. In order to carry out the isolation and integration of this motion engine, two key aspects are important to mention: the implementation of a shared memory block for inter-communicating the motion process directly with NAOs hardware interface (NaoQi-DCM), and the implementation of a blackboard for reading and writing the motion engine inputs and outputs. A basic B-Human actuation block diagram is showed in Figure 2 .

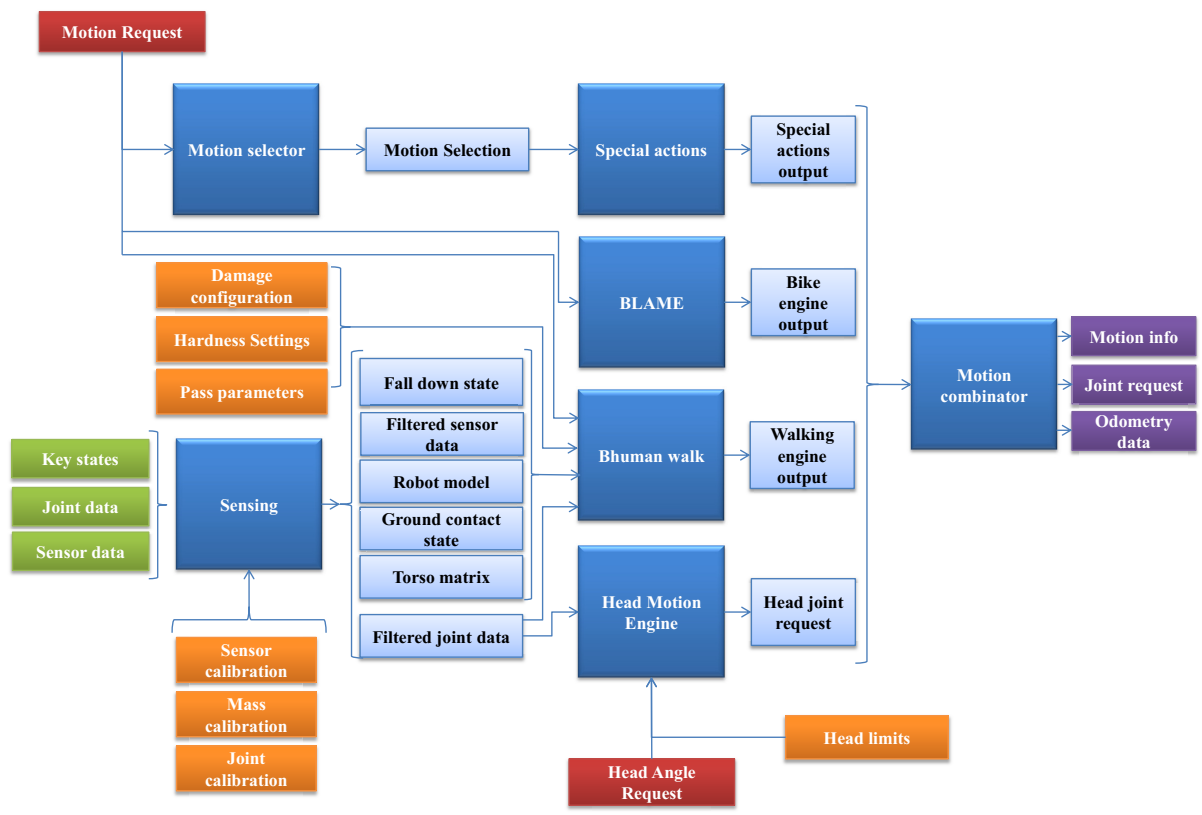

Fig. 2. B-Human actuation block diagram. The blue blocks are the main motion engine modules and the orange blocks some of their configuration parameters. Green blocks are sensors and encoders readings which are the inputs obtained each frame. The outputs (purple blocks) are mainly a joint request and odometric information. Red blocks are the high level requests. (Based on B-Human 2012 team description paper and code release [1]).

In order to integrate the B-Human walking and motion module as a ROS node (bh-motion), several types of mesages have been defined, maintaining as much as possible the compatibility between the original B-Human representations and ROS mesagges. Table 1 shows some of these messages; a complete documentation about their descriptions and functions can be found in [1] and [2].

The basic procedure to subscribe, execute and publish the bh-motion node is as follows: 
Table 1. Some relevant ROS messages defined for the B-Human motion node

\begin{tabular}{|c|c|c|}
\hline Msg. Name & First Level Attributes & Second Level Attributes \\
\hline \multirow{3}{*}{ HeadRequest (input) } & float pan & 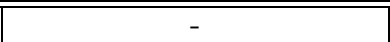 \\
\hline & float tilt & - \\
\hline & float speed & - \\
\hline \multirow{6}{*}{ MotionRequest (input) } & motionType & - \\
\hline & specialActionRequest & $\begin{array}{l}\text { specialActionType } \\
\text { bool mirror }\end{array}$ \\
\hline & & mode \\
\hline & walkRequest & Pose2D speed \\
\hline & & Pose2D target \\
\hline & bikeRequest & $\begin{array}{l}\text { bMotionType } \\
\text { bool mirror }\end{array}$ \\
\hline \multirow{3}{*}{ FilteredJointDataPrev (output) } & float[] angles & - \\
\hline & int timeStamp & - \\
\hline & float cycleTime & - \\
\hline \multirow{3}{*}{ FilteredJointData (output) } & float[] angles & - \\
\hline & int timeStamp & - \\
\hline & float cycleTime & - \\
\hline \multirow{6}{*}{ FilteredSensorData (output) } & float[] data & - \\
\hline & int[] currents & - \\
\hline & uint[] temperatures & - \\
\hline & uint timeStamp & - \\
\hline & uint usActuatorMode & - \\
\hline & uint usTimeStamp & - \\
\hline \multirow{3}{*}{ GroundContactState(output) } & bool contactSafe & - \\
\hline & bool contact & - \\
\hline & bool noContactSafe & - \\
\hline \multirow{4}{*}{ HeadJointRequest (output) } & float tilt & - \\
\hline & float pan & - \\
\hline & bool reachable & - \\
\hline & bool moving & - \\
\hline \multirow{2}{*}{ JointRequest (output) } & jointData & filteredJointData \\
\hline & jointHardness & int][ hardness \\
\hline OdometryData (output) & pose2D & - \\
\hline \multirow{3}{*}{ RobotModel (output) } & Pose3D[] limbs & - \\
\hline & Vector3 centerOfMass & - \\
\hline & float totalMass & - \\
\hline TorsoMatrix (output) & Pose3D offset & - \\
\hline
\end{tabular}

1. The node subscribes to the correspondent ROS topics waiting for a motion request.

2. If there is a callback, the input messages are copied to the blackboard.

3. Every time NaoQi-DCM updates the sensors, the motion engine checks the blackboard for pending jobs and computes the motion engine to obtain its outputs (mainly actuator requests and odometric information). 
4. Obtained outputs are put in the blackboard and published on its correspondent ROS topics.

5. Naoqi-DCM receives the actuator requested values and updates the values of the sensors again.

The source code of bh-motion ROS package can be downloaded in [9], soon it will be shared on the ROS community website.

\section{Proof of Concept}

\subsection{Communication between Two NAO Robots}

The objective of this proof of concept is communicating two robots by using the ROS framework, and its distributed computing capability. The first robot (Robot 1 - Goalie) has the UChile Robotics Team code with an interface for sharing the most important representations as ROS messages (perceptors, odometry, robot pose, etc). The second robot (robot 2 - blind), only has the B-Human motion engine ROS node, and it does not have any vision or perception module.

Robot 1 is the master, it is static, knows its pose on the field and can see the ball. That robot publishes the global position of the seen ball in a topic which is subscribed by blind robot. Robot 2 knows its initial pose on the field, it receives a callback from robot 1 with the ball position, transforms it to an adequate navigation message (walk request), and goes to the ball.

The procedure for sharing the ball position from robot 1 to robot 2 can be described as following:

1. Robot 1 receives a READY flag from Robot 2 .

2. Robot 1 publishes the ball position.

3. Robot 2 disables the READY flag while navigates up to the ball position on the field.

4. Robot 2 enables the READY flag once it is on the indicated position.

5. Go to step 1.

Results of this test can be watched on video in this link [10. The experiment has worked as expected taking into a account the purpose of this proof of concept. No communication failures have been observed neither wrong shared ball positions. Since robot 2 goes to the ball only using its odometry to navigate, reached target positions are inaccurate due to cumulative odometry errors.

Table 2 shows average of CPU and RAM consumption of the NAO V4 (ATOM Z530 1.6GHz CPU and $1 \mathrm{~GB}$ RAM) taken while BHmotion node is running. It can be noticed that average consumption of all ROS processes is about $1 \%$ for CPU and $2.6 \%$ for RAM. 
Table 2. Average of CPU and RAM consumption of the NAO V4

\begin{tabular}{c|c|c|c|c} 
Process & $\begin{array}{c}\text { \%CPU } \\
\text { Avg. }\end{array}$ & $\begin{array}{c}\text { \%CPU } \\
\text { Std.dev. }\end{array}$ & $\begin{array}{c}\text { \%RAM } \\
\text { Avg }\end{array}$ & $\begin{array}{c}\text { \%RAM } \\
\text { Std.dev. }\end{array}$ \\
\hline \hline naoqi-bin & 10.69 & 0.53 & 7.81 & 0.10 \\
\hline nao_hald & 5.10 & 0.29 & 0.30 & 0.00 \\
\hline BHmotion & 3.22 & 0.89 & 0.70 & 0.00 \\
\hline rosout & 0.36 & 0.15 & 0.50 & 0.00 \\
\hline roscore & 0.33 & 0.11 & 1.20 & 0.00 \\
\hline rosmaster & 0.33 & 0.11 & 0.90 & 0.00
\end{tabular}

\section{Conclusions}

This article described the use of the ROS middleware in NAO robots. A guide to cross-compile, install and run ROS onto the CPU of the latest NAO robot was presented. Also, a brief description of how B-Human motion engine was integrated as a ROS node has been presented. In addition, a proof of concept which two NAO robots have been successfully communicated by using ROS framework was presented.

Since this is our first approach towards integration of ROS framework with NAOs and soccer robotics, our main objetive was not to measure or compare the ROS computational-performance into the Atom CPU. This is part of our future work.

A line of work that needs to be explored by the comunity is the integration of the bh-motion node with simulation, visualization, debugging, and other useful tools currently available for ROS.

Acknowledgments. This work was partially funded by FONDECYT under Project Number 1130153. We thank B-Human for their contribution to SPL.

\section{References}

1. Röfer, T., Laue, T., Müller, J., Bartsch, M., Batram, M.J., Böckmann, A., Lehmann, N., Maaß, F., Münder, T., Steinbeck, M., Stolpmann, A., Taddiken, S., Wieschendorf, R., Zitzmann, D.: B-Human Team Report and Code Release (2012), http://www.b-human.de/wp-content/uploads/2012/11/CodeRelease2012.pdf

2. Graf, C., Härtl, A., Röfer, T., Laue, T.: A Robust Closed-Loop Gait for the Standard Platform League Humanoid. In: Zhou, C., Pagello, E., Menegatti, E., Behnke, S., Röfer, T. (eds.) Proceedings of the Fourth Workshop on Humanoid Soccer Robots in Conjunction with the 2009 IEEE-RAS International Conference on $\mathrm{Hu}-$ manoid Robots in Conjunction with the 2009 IEEE-RAS International Conference on Humanoid Robots, Paris, France, pp. 30-37 (2009)

3. Yáñez, J.M., Leottau, L., Cano, P., Mattamala, M., Vidal, A., Celedón, W., Mardones, J., Silva, C., Ruiz-del-Solar, J., UChile, R.T.: Team Description Paper, RoboCup 2013 - Standard Platform League. In: RoboCup 2013: Robot Soccer World Cup XVII Symposium Preproceedings (2013) 
4. Austrian-Kangaroos 2013 Team Description Paper. In: RoboCup 2013: Robot Soccer World Cup XVII Symposium Preproceedings (2013)

5. Gouaillier, D., Hugel, V., Blazevic, P., Kilner, C., Monceaux, J., Lafourcade, P., Marnier, B., Serre, J., Maisonnier, B.: Mechatronic design of NAO humanoid. In: 2009 IEEE Int. Conf. Robot. Autom., pp. 769-774. IEEE (2009), http://ieeexplore. ieee.org/articleDetails . jsp?arnumber=5152516

6. UChile Robotics Team: Ros fuerte cross-compiling and installation for the NAO v4, http://www.ros.org/wiki/nao/Tutorials/Cross-Compiling_NAO-V4

7. Hornung, A., Dornbush, A., Likhachev, M., Bennewitz, M.: Anytime search-based footstep planning with suboptimality bounds. In: 2012 12th IEEE-RAS International Conference on Humanoid Robots (Humanoids), pp. 674-679 (2012)

8. Nao-Robot ROS Stack, http://www.ros.org/wiki/nao_robot

9. UChile Robotics Team: B-Human Motion ROS Package (Source Code), https://github.com/uchile/bh-motion_ros-pkg

10. UChile Robotics Team: Communication between two robots (Video), http://www . youtube . com/watch?v=UgR6JxY1R8A\&f eature=youtu . be 